\title{
Complete Complementary Codes and Generalized Reed-Muller Codes
}

\author{
Chao-Yu Chen, Chung-Hsuan Wang, and Chi-chao Chao
}

\begin{abstract}
Due to ideal autocorrelation and cross-correlation properties, complete complementary codes (CCCs) can be employed in CDMA systems to eliminate the multiple-access interference. In this letter, we propose a direct general construction of CCCs from cosets of the first-order Reed-Muller codes, which includes previous results as a special case. The larger number of CCCs constructed by our method can provide advantages in applications to cellular CDMA systems.
\end{abstract}

Index Terms-Complete complementary codes, Golay complementary sets, Reed-Muller codes, CDMA.

\section{INTRODUCTION}

$\mathbf{T}$ HE concept of complementary pairs was first proposed by Golay [1], and then it was extended to Golay complementary sets (GCSs) in [2], where the aperiodic autocorrelations of all the sequences in a GCS are summed to zero except at zero shift. Later in [3] the complete complementary codes (CCCs) were proposed, which can be regarded as a collection of GCSs with the additional aperiodic cross-correlation property. Since CCCs have these autocorrelation and crosscorrelation properties, they can be applied to multicarrier CDMA (MC-CDMA) systems to eliminate the multiple-access interference (MAI) [4], [5]. Other possibilities include applications to radar systems and cellular OFDM systems.

A connection between complementary sequences and ReedMuller (RM) codes was first proposed in [6]. Then the relationship was extended to GCSs in [7]-[9]. While a recursive construction of CCCs was provided in [10], both in [11] and this letter connections of CCCs and cosets of the first-order RM codes are proposed. However, only second-order cosets are considered in [11]; in addition, every CCC constructed in [11] contains only two distinct GCSs regardless of the order of the CCC and the other GCSs are obtained by reordering the sequences in the two distinct sets. In this letter, we provide a general relationship between CCCs and all the cosets of the first-order RM codes and can also construct CCCs consisting of all distinct GCSs. Furthermore, the construction of CCCs from second-order cosets of the first-order RM codes proposed in [11] can be regarded as a special case of our general construction. Therefore, our result can construct much more CCCs than those in [11], which can provide advantages in applications to cellular CDMA systems.

Manuscript received July 24, 2008. The associate editor coordinating the review of this letter and approving it for publication was V. Stankovic. This work was supported by the National Science Council, Taiwan, R.O.C., under Grant No. NSC 93-2213-E-007-021.

C.-Y. Chen and C.-C. Chao are with the Institute of Communications Engineering, National Tsing Hua University, Hsinchu 30013, Taiwan, R.O.C. (e-mail: d919609@Oz.nthu.edu.tw; ccc@ee.nthu.edu.tw).

C.-H. Wang is with the Department of Communication Engineering, National Chiao Tung University, Hsinchu 30010, Taiwan, R.O.C. (e-mail: chwang@mail.nctu.edu.tw).

Digital Object Identifier 10.1109/LCOMM.2008.081189

\section{Complete Complementary Codes}

Let $\boldsymbol{c}=\left(c_{0}, c_{1}, \ldots, c_{n-1}\right)$ and $\boldsymbol{d}=\left(d_{0}, d_{1}, \ldots, d_{n-1}\right)$ be $\mathbb{Z}_{q}$-valued sequences of length $n$, where $c_{i}$ and $d_{i}$ are in the ring $\mathbb{Z}_{q}=\{0,1, \ldots, q-1\}$. In this letter, we consider the ring $\mathbb{Z}_{q}$ for even $q$. The aperiodic cross-correlation function $\rho(\boldsymbol{c}, \boldsymbol{d} ; u)$ of $\boldsymbol{c}$ and $\boldsymbol{d}$ at displacement $u$ is defined as

$$
\rho(\boldsymbol{c}, \boldsymbol{d} ; u)= \begin{cases}\sum_{k=0}^{n-1-u} \xi^{c_{k+u}-d_{k}}, & 0 \leq u \leq n-1 \\ \sum_{k=0}^{n-1+u} \xi^{c_{k}-d_{k-u},}, & -n+1 \leq u<0\end{cases}
$$

where $\xi=e^{2 \pi j / q}$ is a primitive complex $q$ th root of unity. We also define the aperiodic autocorrelation function $\rho(\boldsymbol{c} ; u)$ of a sequence $\boldsymbol{c}$ at displacement $u$ to be $\rho(\boldsymbol{c} ; u)=\rho(\boldsymbol{c}, \boldsymbol{c} ; u)$.

Definition 1: [2] A set of $N$ sequences $\boldsymbol{c}_{0}, \boldsymbol{c}_{1}, \ldots, \boldsymbol{c}_{N-1}$ of length $n$ is called a GCS of order $N$ if the autocorrelation functions satisfy

$$
\rho\left(\boldsymbol{c}_{0} ; u\right)+\rho\left(\boldsymbol{c}_{1} ; u\right)+\cdots+\rho\left(\boldsymbol{c}_{N-1} ; u\right)= \begin{cases}0, & u \neq 0 \\ N n, & u=0 .\end{cases}
$$

Definition 2: [3] The $N$ sets of $N$ length- $n$ sequences $\left\{\boldsymbol{c}_{0}^{0}, \boldsymbol{c}_{1}^{0}, \ldots, \boldsymbol{c}_{N-1}^{0}\right\}, \ldots,\left\{\boldsymbol{c}_{0}^{N-1}, \boldsymbol{c}_{1}^{N-1}, \ldots, \boldsymbol{c}_{N-1}^{N-1}\right\}$ are called a CCC of order $N$ if every set is a GCS and every two distinct GCSs satisfy the additional ideal cross-correlation property

$\sum_{k=0}^{N-1} \rho\left(\boldsymbol{c}_{k}^{i}, \boldsymbol{c}_{k}^{j} ; u\right)=0$, for any $u ; i, j=0, \ldots, N-1 ; i \neq j$.

One application of CCCs to MC-CDMA systems is to assign different GCSs in the same CCC to different users and send the composing sequences in a GCS on different carriers [5]. The receiver then correlates the signals on different carriers by respective sequences in the GCS, and hence ideal crosscorrelations between different GCSs can eliminate the MAI.

\section{CCCS FROM RM CODES}

Let the $r$ th-order RM code of length $2^{m}$ over $\mathbb{Z}_{2}$ be represented by $\operatorname{RM}(r, m)$ and denote the $2^{m}$-tuple vectors by

$$
\boldsymbol{v}_{i}=(\underbrace{00 \cdots 0}_{2^{i-1}} \underbrace{11 \cdots 1}_{2^{i-1}} \underbrace{00 \cdots 0}_{2^{i-1}} \cdots \underbrace{11 \cdots 1}_{2^{i-1}}), \quad i=1,2, \ldots, m
$$

and $\boldsymbol{v}_{0}=(11 \cdots 1)$ which is the all-one vector. Note that $\boldsymbol{v}_{i}$ defined above has the property that the $j$ th bit of $\boldsymbol{v}_{i}$ is equal to $j_{i}$, where $\left(j_{1}, j_{2}, \ldots, j_{m}\right)$ is the binary representation of $j$ with $j_{1}$ the least significant bit. $\operatorname{RM}(r, m)$ is a binary linear code generated by the generator matrix [12] $G_{\mathrm{RM}}(r, m)=$ $\left[\boldsymbol{v}_{0}^{T}, \boldsymbol{v}_{1}^{T}, \boldsymbol{v}_{2}^{T}, \ldots, \boldsymbol{v}_{m}^{T},\left(\boldsymbol{v}_{1} \boldsymbol{v}_{2}\right)^{T}, \ldots,\left(\boldsymbol{v}_{m-1} \boldsymbol{v}_{m}\right)^{T}, \ldots\right.$, up to products of degree $r]^{T}$, where the product of vectors corresponds to the component-wise product. The generalized $r$ thorder RM code [7] of length $2^{m}$, denoted by $\mathrm{RM}_{q}(r, m)$, is defined as the linear code over $\mathbb{Z}_{q}$ generated by the 
same generator matrix as that of the binary RM code. It is straightforward to obtain that any codeword in $\mathrm{RM}_{q}(r, m)$ can be uniquely expressed as a linear combination of rows of $G_{\mathrm{RM}}(r, m)$ over $\mathbb{Z}_{q}$ with operations modulo $q$. The following theorem provides a direct general construction of CCCs from cosets of the first-order RM codes, where $\mathbb{N}_{m}=\{1,2, \ldots, m\}$ and $\boldsymbol{v}_{i}^{0}=\boldsymbol{v}_{0}, \boldsymbol{v}_{i}^{1}=\boldsymbol{v}_{i}$ for $\boldsymbol{v}_{i}$ defined above.

Theorem 1: For any even integer $q$, any positive integer $m$, and any positive integer $k \leq m$, let nonempty sets $I_{1}, I_{2}, \ldots, I_{k}$ be a partition of $\mathbb{N}_{m}, m_{\alpha}=\left|I_{\alpha}\right|$, and $\pi_{\alpha}$ be a bijection from $\mathbb{N}_{m_{\alpha}}$ to $I_{\alpha}$ for $\alpha=1,2, \ldots, k$. Also let

$$
\begin{aligned}
\boldsymbol{Q}= & \frac{q}{2} \sum_{\alpha=1}^{k} \sum_{\beta=1}^{m_{\alpha}-1} \boldsymbol{v}_{\pi_{\alpha}(\beta)} \boldsymbol{v}_{\pi_{\alpha}(\beta+1)} \\
& +\sum_{\alpha=2}^{k} \sum_{\beta=1}^{m_{\alpha}} \sum_{\tau=0}^{2^{\alpha-1}-1} \lambda_{\alpha, \beta, \tau} \boldsymbol{v}_{\pi_{\alpha}(\beta)} \prod_{\gamma=1}^{\alpha-1} \boldsymbol{v}_{\pi_{\gamma}\left(m_{\gamma}\right)}^{\tau_{\gamma}}
\end{aligned}
$$

where $\lambda_{\alpha, \beta, \tau} \in \mathbb{Z}_{q}$ and $\left(\tau_{1}, \tau_{2}, \ldots, \tau_{\alpha-1}\right)$ is the binary representation of $\tau$. For any codeword $\boldsymbol{c} \in \boldsymbol{Q}+\mathrm{RM}_{q}(1, m)$ and for $p, n=0,1, \ldots, 2^{k}-1$, if we let

$$
\boldsymbol{c}_{n}^{p}=\boldsymbol{c}+\frac{q}{2} \sum_{\alpha=1}^{k} n_{\alpha} \boldsymbol{v}_{\pi_{\alpha}(1)}+\frac{q}{2} \sum_{\alpha=1}^{k} p_{\alpha} \boldsymbol{v}_{\pi_{\alpha}\left(m_{\alpha}\right)}
$$

where $\left(n_{1}, n_{2}, \cdots, n_{k}\right)$ and $\left(p_{1}, p_{2}, \cdots, p_{k}\right)$ are the binary representations of $n$ and $p$, respectively, and let $G^{p}=$ $\left\{\boldsymbol{c}_{0}^{p}, \boldsymbol{c}_{1}^{p}, \ldots, \boldsymbol{c}_{2^{k}-1}^{p}\right\}$, then $G^{0}, G^{1}, \ldots, G^{2^{k}-1}$ form a CCC of order $2^{k}$ and length $2^{m}$.

Proof: In the first part, since $\rho(\boldsymbol{d} ; u)=\rho^{*}(\boldsymbol{d} ;-u)$ for any sequence $\boldsymbol{d}$, to demonstrate that every $G^{p}$ is a GCS of order $2^{k}$ for $p=0,1, \ldots, 2^{k}-1$, we have to show that for $u>0$,

$$
\sum_{\boldsymbol{d} \in G^{p}} \sum_{i=0}^{2^{m}-1-u} \xi^{d_{i+u}-d_{i}}=\sum_{i=0}^{2^{m}-1-u} \sum_{\boldsymbol{d} \in G^{p}} \xi^{d_{i+u}-d_{i}}=0 .
$$

For any integer $i$, let $j=i+u$; also let $\left(i_{1}, i_{2}, \ldots, i_{m}\right)$ and $\left(j_{1}, j_{2}, \ldots, j_{m}\right)$ be the binary representations of $i$ and $j$, respectively.

Case 1: If $i_{\pi_{\alpha}(1)} \neq j_{\pi_{\alpha}(1)}$ for some $\alpha \in\{1,2, \ldots, k\}$, then for any sequence $\boldsymbol{d} \in G^{p}$, there exists $\boldsymbol{d}^{\prime}=$ $\left(d_{0}^{\prime}, d_{1}^{\prime}, \ldots, d_{2^{m}-1}^{\prime}\right)=\boldsymbol{d}+(q / 2) \boldsymbol{v}_{\pi_{\alpha}(1)} \in G^{p}$ such that

$d_{j}-d_{i}-d_{j}^{\prime}+d_{i}^{\prime}=\frac{q}{2}\left(i_{\pi_{\alpha}(1)}-j_{\pi_{\alpha}(1)}\right) \equiv \frac{q}{2} \quad(\bmod q)$.

So we have $\xi^{d_{j}-d_{i}} / \xi^{d_{j}^{\prime}-d_{i}^{\prime}}=\xi^{q / 2}=-1$ which implies $\xi^{d_{j}-d_{i}}+\xi^{d_{j}^{\prime}-d_{i}^{\prime}}=0$. Hence, we have

$$
\sum_{\boldsymbol{d} \in G^{p}} \xi^{d_{i+u}-d_{i}}=0
$$

Case 2: In this case, we have $i_{\pi_{\alpha}(1)}=j_{\pi_{\alpha}(1)}$ for all $\alpha=$ $1,2, \ldots, k$. Suppose that $i_{\pi_{\alpha}(\beta)}=j_{\pi_{\alpha}(\beta)}$ for $\alpha=1,2, \ldots, \hat{\alpha}-$ $1, \beta=1,2, \ldots, m_{\alpha}$ and $\hat{\beta}$ is the smallest integer such that

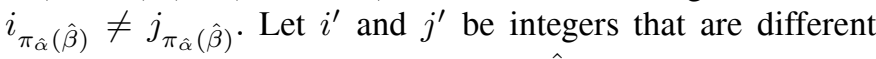
from $i$ and $j$ in only one position $\pi_{\hat{\alpha}}(\hat{\beta}-1)$, i.e., $i_{\pi_{\hat{\alpha}}(\hat{\beta}-1)}^{\prime}=$ $1-i_{\pi_{\hat{\alpha}}(\hat{\beta}-1)}$ and $j_{\pi_{\hat{\alpha}}(\hat{\beta}-1)}^{\prime}=1-j_{\pi_{\hat{\alpha}}(\hat{\beta}-1)}$, respectively, and so $j^{\prime}=i^{\prime}+u$. For any sequence $\boldsymbol{d} \in G^{p}, \boldsymbol{d}$ can be expressed as $\boldsymbol{d}=\boldsymbol{Q}+\sum_{l=0}^{m} g_{l} \boldsymbol{v}_{l}$ where $g_{l} \in \mathbb{Z}_{q}$, and then we can obtain

$$
\begin{aligned}
& d_{i^{\prime}}-d_{i}=\frac{q}{2}\left(i_{\pi_{\hat{\alpha}(\hat{\beta}-2)}} i_{\pi_{\hat{\alpha}(\hat{\beta}-1)}^{\prime}}-i_{\pi_{\hat{\alpha}(\hat{\beta}-2)}} i_{\pi_{\hat{\alpha}}(\hat{\beta}-1)}\right. \\
& +i_{\pi_{\hat{\alpha}(\hat{\beta}-1)}^{\prime}} i_{\pi_{\hat{\alpha}(\hat{\beta})}}-i_{\pi_{\hat{\alpha}(\hat{\beta}-1)}} i_{\left.\pi_{\hat{\alpha}(\hat{\beta})}\right)} \\
& +\sum_{\tau=0}^{2^{\hat{\alpha}-1}-1} \lambda_{\hat{\alpha}, \hat{\beta}-1, \tau} i_{\pi_{\hat{\alpha}}(\hat{\beta}-1)} \prod_{\gamma=1}^{\hat{\alpha}-1} i_{\pi_{\gamma}\left(m_{\gamma}\right)}^{\tau_{\gamma}} \\
& -\sum_{\tau=0}^{2^{\hat{\alpha}-1}-1} \lambda_{\hat{\alpha}, \hat{\beta}-1, \tau} i_{\pi_{\hat{\alpha}}(\hat{\beta}-1)} \prod_{\gamma=1}^{\hat{\alpha}-1} i_{\pi_{\gamma}\left(m_{\gamma}\right)}^{\tau_{\gamma}}
\end{aligned}
$$

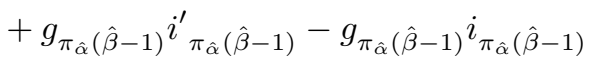

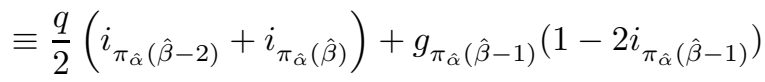

$$
\begin{aligned}
& +\sum_{\tau=0}^{2^{\hat{\alpha}-1}-1} \lambda_{\hat{\alpha}, \hat{\beta}-1, \tau}\left(1-2 i_{\pi_{\hat{\alpha}}(\hat{\beta}-1)}\right) \prod_{\gamma=1}^{\hat{\alpha}-1} i_{\pi_{\gamma}\left(m_{\gamma}\right)}^{\tau_{\gamma}} \quad(\bmod q)
\end{aligned}
$$

where the first equality follows from (1) and the condition that $i$ and $i^{\prime}$ differ in only one position $\pi_{\hat{\alpha}}(\hat{\beta}-1)$. Note that we assume $\hat{\beta} \geq 3$ here. For the case $\hat{\beta}=2$, we can just remove those terms involving $\hat{\beta}-2$ in the preceding equation. Since $i_{\pi_{\hat{\alpha}}(\hat{\beta}-2)}=j_{\pi_{\hat{\alpha}(\hat{\beta}-2)}}, i_{\pi_{\hat{\alpha}(\hat{\beta}-1)}}=j_{\pi_{\hat{\alpha}(\hat{\beta}-1)}}$, and $i_{\pi_{\gamma}\left(m_{\gamma}\right)}=$ $j_{\pi_{\gamma}\left(m_{\gamma}\right)}$ for $\gamma=1,2, \ldots, \hat{\alpha}-1$, we have

$$
d_{j}-d_{i}-d_{j^{\prime}}+d_{i^{\prime}} \equiv \frac{q}{2}\left(i_{\pi_{\hat{\alpha}(\hat{\beta})}}-j_{\pi_{\hat{\alpha}}(\hat{\beta})}\right) \equiv \frac{q}{2} \quad(\bmod q)
$$

which implies $\xi^{d_{j}-d_{i}}+\xi^{d_{j^{\prime}}-d_{i^{\prime}}}=0$. Hence, we have

$$
\sum_{\boldsymbol{d} \in G^{p}} \xi^{d_{i+u}-d_{i}}+\xi^{d_{i^{\prime}+u}-d_{i^{\prime}}}=0 .
$$

Combining these two cases, we can obtain that $G^{p}$ is a GCS for all $p=0,1, \ldots, 2^{k}-1$.

Then, in the second part, we will show that any two distinct sets $G^{s}$ and $G^{t}$ where $0 \leq s \neq t \leq 2^{k}-1$ satisfy the ideal cross-correlation property. For $u>0$, we have to show

$$
\sum_{n=0}^{2^{k}-1} \sum_{i=0}^{2^{m}-1-u} \xi^{c_{n, i+u}^{s}-c_{n, i}^{t}}=\sum_{i=0}^{2^{m}-1-u} \sum_{n=0}^{2^{k}-1} \xi^{c_{n, i+u}^{s}-c_{n, i}^{t}}=0
$$

where we denote $c_{n}^{p}=\left(c_{n, 0}^{p}, c_{n, 1}^{p}, \ldots, c_{n, 2^{m}-1}^{p}\right)$ for $p=s, t$ and $n=0,1, \ldots, 2^{k}-1$. Similarly, for any integer $i$, let $j=i+u$.

Case 1: If $i_{\pi_{\alpha}(1)} \neq j_{\pi_{\alpha}(1)}$ for some $\alpha \in\{1,2, \ldots, k\}$, then for sequences $c_{n}^{p}=\left(c_{n, 0}^{p}, c_{n, 1}^{p}, \ldots, c_{n, 2^{m}-1}^{p}\right) \in G^{p}, p=$ $s, t$, there exist sequences $c_{n^{\prime}}^{p}=\left(c_{n^{\prime}, 0}^{p}, c_{n^{\prime}, 1}^{p}, \ldots, c_{n^{\prime}, 2^{m}-1}^{p}\right)=$ $\boldsymbol{c}_{n}^{p}+(q / 2) \boldsymbol{v}_{\pi_{\alpha}(1)} \in G^{p}$ such that $c_{n, j}^{s}-c_{n, i}^{t}-c_{n^{\prime}, j}^{s}+c_{n^{\prime}, i}^{t}=$ $(q / 2)\left(i_{\pi_{\alpha}(1)}-j_{\pi_{\alpha}(1)}\right) \equiv q / 2(\bmod q)$. So, similar to Case 1 of the first part, we have $\sum_{n=0}^{2^{k}-1} \xi^{c_{n, i+u}^{s}-c_{n, i}^{t}}=0$.

Case 2: In this case, we have $i_{\pi_{\alpha}(1)}=j_{\pi_{\alpha}(1)}$ for all $\alpha=$ $1,2, \ldots, k$. Let $\hat{\alpha}, \hat{\beta}, i^{\prime}$, and $j^{\prime}$ be given as in Case 2 of the first part. We can also obtain that $c_{n, j}^{s}-c_{n, i}^{t}-c_{n, j^{\prime}}^{s}+c_{n, i^{\prime}}^{t} \equiv q / 2$ $(\bmod q)$, and hence $\sum_{n=0}^{2^{k}-1} \xi^{c_{n, i+u}^{s}-c_{n, i}^{t}}+\xi^{c_{n, i^{\prime}+u}^{s}-c_{n, i^{\prime}}^{t}}=0$.

From Cases 1 and 2, we can obtain that (2) holds for $u>0$. Similarly, it can also be obtained that $\sum_{n=0}^{2^{k}-1} \sum_{i=0}^{2^{m}-1+u} \xi^{c_{n, i}^{s}-c_{n, i-u}^{t}}=0$ for $u<0$. Now, it remains 
TABLE I

COMPARISON OF $\Delta(k, m)$ AND $\Delta_{\text {New }}(k, m)$ FOR $m=4$

\begin{tabular}{|c|c|c|c|c|}
\hline$k$ & 1 & 2 & 3 & 4 \\
\hline Orders of CCCs $\left(2^{k}\right)$ & 2 & 4 & 8 & 16 \\
\hline$\Delta(k, 4)$ & 12 & 49 & 63 & 64 \\
\hline$\Delta_{\text {new }}(k, 4)$ & 12 & 52 & 479 & 2048 \\
\hline
\end{tabular}

to show that

$$
\sum_{n=0}^{2^{k}-1} \rho\left(\boldsymbol{c}_{n}^{s}, \boldsymbol{c}_{n}^{t} ; 0\right)=\sum_{n=0}^{2^{k}-1} \sum_{i=0}^{2^{m}-1} \xi^{c_{n, i}^{s}-c_{n, i}^{t}}=0 .
$$

For any nonnegative integer $n<2^{k}$, we have $\boldsymbol{c}_{n}^{s}-\boldsymbol{c}_{n}^{t} \equiv$ $(q / 2) \boldsymbol{d}(\bmod q)$ where $\boldsymbol{d}=\left(s_{1} \oplus t_{1}\right) \boldsymbol{v}_{\pi_{1}\left(m_{1}\right)} \oplus\left(s_{2} \oplus\right.$ $\left.t_{2}\right) \boldsymbol{v}_{\pi_{2}\left(m_{2}\right)} \oplus \cdots \oplus\left(s_{k} \oplus t_{k}\right) \boldsymbol{v}_{\pi_{k}\left(m_{k}\right)}$ and $\oplus$ denotes mod-2 addition; $\left(s_{1}, s_{2}, \ldots, s_{k}\right)$ and $\left(t_{1}, t_{2}, \ldots, t_{k}\right)$ are the binary representations of $s$ and $t$, respectively. It can be easily obtained that the Hamming weight of $\boldsymbol{d}$ is $2^{m-1}$. Hence, for $i=0,1, \ldots, 2^{m}-1$, there are $2^{m-1}$ pairs $\left(c_{n, i}^{s}, c_{n, i}^{t}\right)$ such that $\xi^{c_{n, i}^{s}-c_{n, i}^{t}}=\xi^{q / 2}=-1$ and $2^{m-1}$ pairs $\left(c_{n, i}^{s}, c_{n, i}^{t}\right)$ such that $\xi^{c_{n, i}^{s}-c_{n, i}^{t}}=\xi^{0}=1$. So we have $\rho\left(\boldsymbol{c}_{n}^{s}, \boldsymbol{c}_{n}^{t} ; 0\right)=$ $\sum_{i=0}^{2^{m}-1} \xi^{c_{n, i}^{s}-c_{n, i}^{t}}=0$, which completes the proof.

Example 1: For $q=2, m=6$, and $k=3$, we let $I_{1}=$ $\{1,2\}, I_{2}=\{3,4\}, I_{3}=\{5,6\}, \pi_{1}(1)=1, \pi_{1}(2)=2$, $\pi_{2}(1)=3, \pi_{2}(2)=4, \pi_{3}(1)=5$, and $\pi_{3}(2)=6$. If we denote $\boldsymbol{Q}=\sum_{\alpha=1}^{3} \sum_{\beta=1}^{1} \boldsymbol{v}_{\pi_{\alpha}(\beta)} \boldsymbol{v}_{\pi_{\alpha}(\beta+1)}+\boldsymbol{v}_{\pi_{3}(2)} \prod_{\gamma=1}^{2} \boldsymbol{v}_{\pi_{\gamma}(2)}=$ $\boldsymbol{v}_{1} \boldsymbol{v}_{2}+\boldsymbol{v}_{3} \boldsymbol{v}_{4}+\boldsymbol{v}_{5} \boldsymbol{v}_{6}+\boldsymbol{v}_{6} \boldsymbol{v}_{2} \boldsymbol{v}_{4}$, then for any codeword $\boldsymbol{c}$ in this third-order coset $\boldsymbol{Q}+\mathrm{RM}(1,6)$, we have that the sets $C^{p}=\left\{\boldsymbol{c}+p_{1} \boldsymbol{v}_{2}+p_{2} \boldsymbol{v}_{4}+p_{3} \boldsymbol{v}_{6}+n_{1} \boldsymbol{v}_{1}+n_{2} \boldsymbol{v}_{3}+n_{3} \boldsymbol{v}_{5}:\right.$ $\left.n_{i} \in \mathbb{Z}_{2}\right\}$ for $p=0,1, \ldots, 7$, where $\left(p_{1}, p_{2}, p_{3}\right)$ is the binary representation of $p$, form a CCC of order 8 and length 64 . Note that these 8 GCSs are all distinct.

From Theorem 1, we know that every sequence in the coset $\boldsymbol{Q}+\mathrm{RM}_{q}(1, m)$ lies in a CCC and hence the coset consists of several CCCs. If $m_{\alpha} \geq 2$ for all $\alpha=1,2, \ldots, k$, then it can be found that our constructed CCCs of order $2^{k}$ comprises $2^{k}$ all distinct GCSs while the CCCs constructed in [11] contains only two distinct GCSs, regardless of the order $2^{k}$, and the other GCSs are obtained by reordering the sequences in the two distinct GCSs. The coset representatives $Q$ given in (1) can cover all the cosets of $\operatorname{RM}_{q}(1, m)$ while only the secondorder cosets are considered in [11]. Furthermore, if we set $m_{\alpha}=1$ for $\alpha=1,2, \ldots, k-1$ in Theorem 1 and the coset representatives $\boldsymbol{Q}$ given in (1) are restricted to secondorders, then the coset representatives $Q$ can be reduced to those proposed in [11].

We denote the numbers of cosets of $\operatorname{RM}(1, m)$ which consists of CCCs of order $2^{k}$ and can be derived by the construction in [11] and our construction by $\Delta(k, m)$ and $\Delta_{\text {new }}(k, m)$, respectively. As an example shown in Table I, it can be found that $\Delta_{\text {new }}(k, m)$ is much larger than $\Delta(k, m)$ when $k$ increases since our construction can contain highorder cosets of $\operatorname{RM}(1, m)$ while only second-order cosets are considered in [11].

Note that when CCCs are applied to cellular CDMA systems, MAI can be eliminated within a cell since each user within a cell is assigned a different GCS in the same CCC [5]. Hence in order to serve a large number of users within a cell, a CCC of a large order is desired. Furthermore, different CCCs should be employed for adjacent cells, and hence it is required that GCSs assigned in adjacent cells should have low crosscorrelations to achieve low adjacent-cell interference. Since we can construct much more CCCs than those in [11], we can have more candidates of CCCs for use in adjacent cells to obtain low cross-correlations. For example, for order 8 and length 16, from our construction we can find two binary CCCs of which the largest sum of mutual cross-correlations of two different GCSs in the two CCCs over all shifts is 32 , of which the largest possible value could be $8 \cdot 16^{2}=2048$ for any two sets of order 8 and length 16 , while that of CCCs constructed in [11] is 64 .

\section{CONCLUding Remark}

In this letter, we provide a general connection between CCCs and cosets of first-order RM codes, which includes the results in [11] as a special case. Besides applications to cellular CDMA systems, due to ideal autocorrelation/crosscorrelation properties and low peak-to-average power ratios [6]-[9], one possible further application of CCCs could be to replace pseudo-noise sequences as preamble sequences in cellular OFDM systems [13].

\section{REFERENCES}

[1] M. J. E. Golay, "Complementary series," IEEE Trans. Inform. Theory, vol. IT-7, pp. 82-87, Apr. 1961.

[2] C.-C. Tseng and C. L. Liu, "Complementary sets of sequences," IEEE Trans. Inform. Theory, vol. IT-18, pp. 644-652, Sept. 1972.

[3] N. Suehiro and M. Hatori, " $N$-shift cross-orthogonal sequences," IEEE Trans. Inform. Theory, vol. 34, pp. 143-146, Jan. 1988.

[4] S.-M. Tseng and M. R. Bell, "Asynchronous multicarrier DS-CDMA using mutually orthogonal complementary sets of sequences," IEEE Trans. Commun., vol. 48, pp. 53-59, Jan. 2000.

[5] H.-H. Chen, J.-F. Yeh, and N. Suehiro, "A multicarrier CDMA architecture based on orthogonal complete complementary codes for new generations of wideband wireless communications," IEEE Commun. Mag., vol. 39, pp. 126-134, Oct. 2001.

[6] J. A. Davis and J. Jedwab, "Peak-to-mean power control in OFDM, Golay complementary sequences, and Reed-Muller codes," IEEE Trans. Inform. Theory, vol. 45, pp. 2397-2417, Nov. 1999.

[7] K. G. Paterson, "Generalized Reed-Muller codes and power control in OFDM modulation," IEEE Trans. Inform. Theory, vol. 46, pp. 104-120, Jan. 2000

[8] K.-U. Schmidt, "Complementary sets, generalized Reed-Muller codes, and power control for OFDM," IEEE Trans. Inform. Theory, vol. 53, pp. 808-814, Feb. 2007.

[9] C.-Y. Chen, C.-H. Wang, and C.-C. Chao, "Complementary sets and Reed-Muller codes for peak-to-average power ratio reduction in OFDM," in Proc. 16th Int. Symp. AAECC, LNCS 3857, Las Vegas, NV, Feb. 2006, pp. 317-327.

[10] R. Appuswamy and A. K. Chaturvedi, "A new framework for constructing mutually orthogonal complementary sets and ZCZ sequences," IEEE Trans. Inform. Theory, vol. 52, pp. 3817-3826, Aug. 2006.

[11] A. Rathinakumar and A. K. Chaturvedi, "Complete mutually orthogonal Golay complementary sets from Reed-Muller codes," IEEE Trans. Inform. Theory, vol. 54, pp. 1339-1346, Mar. 2008.

[12] S. Lin and D. J. Costello, Jr., Error Control Coding. 2nd ed. Upper Saddle River, NJ: Pearson Prentice Hall, 2004.

[13] C.-Y. Chen, Y.-J. Min, K.-Y. Lu, and C.-C. Chao, "Cell search for cellbased OFDM systems using quasi complete complementary codes," in Proc. IEEE Int. Conf. Commun., Beijing, China, May 2008, pp. 48404844. 